\title{
用热解化学气沉积法选择性生长 金刚石薄膜的研究
}

\author{
于三金曾孙 吕宪义 邻广田 \\ (吉林大学原子与分子物理研究所,长春 130023)
}

关链词金刚石薄膜、选择性生长、灯丝热解 CVD 方法

\section{一、引 盲}

金刚石薄膜作为一种新型多功能材料, 其制备和应用研究在近几年内取得了飞速的发展. 目前, 用各种化学气相沉积方法 (CVD) 合成的金刚石薄膜在一些领域内已取得了初步的应 用 ${ }^{[山-3]}$. 金刚石薄膜的选择性生长就是在补底表面上按照所需图形生长金刚石薄膜, 因此选择 性生长金刚石薄膜在大规模集成电路、半导体微波器件、半导体光电器件等半导体器件的制备 中作为隔离和散热介质具有广阔的应用前景.

1988 年, 日本的 Hirabayashi 等人年首先用光刻技术和 $\mathrm{Ar}^{+}$离子湤射的祄底表 面 处理 方法在单晶硅衬底上实现了金刚石薄膜的选择性生长. 这一成果引起了人们的极大兴趣和重 视.

本文报道了用灯丝热解化学气相沉积法在经光刻、腐蚀及 $\mathrm{SiO}_{2}$ 薄膜掩盖等方法处理的 硅祄底表面上选择性生长金刚石薄膜的方法和结果, 并对其生长特性进行了讨论.

\section{二、实验制 备}

本文采用灯丝热解 CVD 方法合成选择性生长金刚石薄膜; 其装置与文献 [5]所报道的相 同. 选择性生长金刚石薄膜的合成条件与一般的金刚石薄膜的制备条件大体相同, 所采用的 原料气体是一定比例的甲烷和氢气混合气体, 甲烷浓度为 $0.2 \%$ (体积比); 衬底材料为单晶硅 片 (111); 反应压力为 $4 \mathrm{kPa}$; 衬底温度为 $800-1000^{\circ} \mathrm{C}$; 灯丝与祄底表面的距离为 $10 \mathrm{~mm}$; 合 成时间为 $5 \mathrm{~h}$.

实现金刚石薄膜的选择性生长的关键是对补底表面的处理, 实验上我们采用两种不同的 讨底表面处理方法制备选择性生长金刚石薄膜.

第一种衬底表面处理方法是先用 $\phi 0.5 \mu \mathrm{m}$ 的金刚石研磨离对抛光的单晶硅衬底表面研磨 半小时, 再用光刻方法在研磨过的硅祄底表面按照一定图形用光刻胶进行保护, 然后用一定比 例的 $\mathrm{HF}$ 和 $\mathrm{HNO}_{3}$ 腐蚀液对未用光刻胶保护的研磨过的单晶硅衬底表面进行腐蚀, 最后除 去光刻胶. 用这种方法处理过的硅衬底表面, 在用光刻胶保护的部位仍保持原来的研磨表面 状态; 而其它部位经腐蚀后其表面状态发生变化且具有一定深度的凹䧄. 通过适当控制合成

本文 1990 年 3 月 2 日收到. 
条件金刚石膜只形成于具有原研磨表面状态的祄底表面, 而其它部位表面上只形成一定密度 的分立的金刚石颗粒. 图 1 表示用上述方法处理的祄底表面上选择性生长的金刚石薄膜的扫 描电镜照片. 由图可见,金刚石薄膜只按照预先设计的图案生长,而不需要生长金刚石薄膜的 地方只形成分立的金刚石颗粒。

第二种村底表面处理方法是先用 $\phi 0.5 \mu \mathrm{m}$ 的金刚石研磨育对抛光的单晶磪祄底表面研磨 半小时, 然后用 CVD 方法在研磨过的祄底表面上沉积一层 $\mathrm{SiO}_{2}$ 膜,用光刻方法在 $\mathrm{SiO}_{2}$ 膜 上按设计图形用光刻胶保护; 然后用一定浓度的 $\mathrm{HF}$ 溶液腐蚀除去未经光刻胶保护的 $\mathrm{SiO}_{2}$ 膜, 除去光刻胶,化学清洗. 用这种方法处理的衬底表面, 在用光刻胶保护的地方是 $\mathrm{SiO}_{2}$ 膜, 其它地方为原来的研磨硅祄底表面. 适当控制合成条件使金刚石只生长在研磨的硅衬底表面 上,而在 $\mathrm{SiO}_{2}$ 膜表面几乎不生长或只生长数量极少的分立的金刚石颗粒. 最后用一定浓度 的 $\mathrm{HF}$ 溶液腐蚀除掉硅表面的 $\mathrm{SiO}_{2}$ 膜及生长于其上的分立的金刚石颗粒得到完备的选择性 生长金刚石薄膜的结果. 图 2 为用该方法处理的祄底表面上生长的选择性金刚石薄膜的扫描 电镜照片。



图 1 在光刻和化学扈蚀方法处理的 硅 祄底上制备的选择性生长金咪石薄膜的 扫描电镜照片

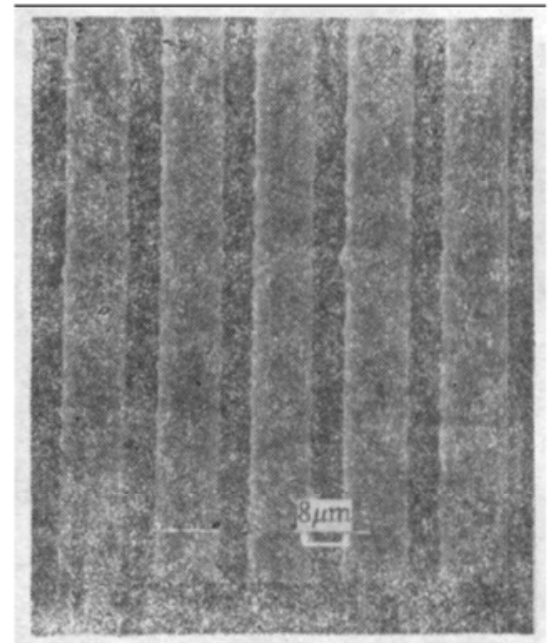

图 2 在 $\mathrm{SiO}_{2}$ 膜覆盖和光刻方法处理的 硅祄底上制备的选择性生长的金刚石薄 瞙的扫描电镜照片

\section{三、结果 讨 论}

金刚石薄膜的选择性生长是通过对衬底表面的特殊处理以及适当控制制备参数，使在祄 底表面不同部位上的金刚石的成核密度出现较大差别而实现的.

实验结果表明,金刚石的成核密度主要与祄底表面状态、祄底表面材料有关. 在本文的实 验条件下实验中观察到的金刚石在 $\mathrm{SiO}_{2}$ 膜表面和各种硅表面上的成核密度列于 表 1 , 这里 所给出的成核密度的数据是根据扫描电子显微镜及光学显微镜对合成的金刚石晶粒数的观测 结果而得到的,因此仅是个数量级的估计.

表 1 金刚石的成核密度与祄底表面材料及祄底表面状态的关系

\begin{tabular}{|c|c|c|c|c|}
\hline 村底表面 & 执光硅表面 & 研楽硅表面 & 碑蚀硅表面 & $\mathrm{SiO}_{2}$ 腽表面 \\
\hline 成核密度 $\left(\mathrm{cm}^{-2}\right)$ & $<10^{3}$ & $>10^{\prime}$ & $<10^{+}$ & $<10^{2}$ \\
\hline
\end{tabular}


本文的第一种选择性生长金刚石薄膜的方法就是利用同种祄底枌料不同衬底表面状忘下 金刚石的成核密度的差别而实现金刚石薄膜的选择性生长的. 实验发现, 抛光的硅祄底表面 经金刚石研磨育研磨后, 金刚石的成核密度可以提高几个数量级, 而对研磨过的硅祄底表面再 用一定的酸溶液腐蚀后反而会使金刚石的成核密度大大降低. 因此, 用这种方法制备的选择 性金刚石薄膜, 金刚石在研磨的祄底表面上按照一定图形形成连续的多晶薄膜, 同时在腐蚀过 的硅表面也形成分立的金刚石颗粒, 由此可见，用该方法制备的选择性金刚石薄膜具有一些明 显的缺点,其一是在酸腐蚀过的祄底表面上生长的分立的金刚石颗粒很难去掉; 其二是由于酸 腐蚀,使衬底表面凹陷, 也就是说衬底表面出现一定程度的破坏, 这对于选择性金刚石膜的实 际应用是相当不利的. 为了克服上述缺点, 我们又采用了第二种方法得到了完备的选择性生长 金刚石薄膜的结果. 通过一系列的实验, 我们发现在适当的制备条件下, 在研磨过的硅表面和 $\mathrm{SiO}_{2}$ 膜表面上金刚石的成核密度具有很大差别, 而且在不破坏衬底表面的情况下, 硅祄底上 的 $\mathrm{SiO}_{2}$ 膜用 $\mathrm{HF}$ 酸很容易除去, 此外硅祄底表面上的 $\mathrm{SiO}_{2}$ 膜也很容易制备. 因此, 用本文 所报道的在硅衬底表面预先生长 $\mathrm{SiO}_{2}$ 膜和光刻方法处理硅祄底表面, 然后生长全刚石, 最后 席蚀除去 $\mathrm{SiO}_{2}$ 膜及生长于其上的金刚石颗㕸的方法在单晶硅褯上能够制备出完备的选 择 性生长金刚石薄膜,而且衬底表面未被破坏.

用热解化学气相沉积方法制备选择性生长金刚石薄膜, 由于其选择性生长图形可以人为 地控制, 金刚石膜本身具有高导热、高绝缘等优异性能, 因此用这种方法制备的选择性金刚石 薄膜在大规模集成电路、半导微波器件、半导体光电器件等半导体器件的制备中作为隔离或散 热材料具有广阔的应用前景. 目前, 各国都在深人开展金刚石薄膜在电子学方面的应用研究, 发展金刚石薄膜的新的制备技术,其中包括选择性生长.

本文的结果表明,采用 $\mathrm{SiO}_{2}$ 薄膜覆盖方法,利用灯丝热解 CVD 设备在单晶硅衬底上按 照实用的要求可制备出具有一定图形的完备的选择性生长的金刚石薄膜, 而且对祄底本身无 任何破坏, 这对于选择性生长全刚石薄膜的实际应用具有十分重要的意义.

\section{参文献}

[1] Ohno, T., Shohata, N., New Diamond (Japan New Diamond Forum), $1988,49$.

[2] Okuzumi, F., New Diamond (Japan New Diamond Forum), 1988, 64.

[3] Yazu, S., New Diamond(Japan New Diamond Forum), 1988, 62.

[4] Harabayashi, K., Taniguchi, Y., Takamatsu, O. et al., Appl. Phys. Lett., 53(1988), 1815.

[5] 金暗孙、品宪义、曲承林等,吉林大学自然科学学报, 2(1987), 127. 